American Journal of Applied Sciences 3 (10): 2033-2036, 2006

ISSN 1546-9239

(c) 2006 Science Publications

\title{
Effect of Magnetic Field on the Formation of Spin-polaron in Mn-Zn Ferrites
}

\author{
H. M. El-Sayed \\ Department of Physics, Faculty of Science, Ain Shams University \\ 11655, Abbassia, Cairo, Egypt
}

\begin{abstract}
The effect of magnetic field on the transport properties of Mn- $\mathrm{Zn}$ ferrite was studied. It was found that, the resistivity has an inverse relation with the applied magnetic field. This was attributed to the reduction of the scattering between the charge carriers and the disordered spins. Also, it was found that, the activation energy in the paramagnetic region is strongly affected by the applied magnetic field. These results are explained in light view of the spin polaron formation and its effect on the effective mass of the charge carriers.
\end{abstract}

Key words: Mn-Zn ferrites, transport properties, magnetic spin polaron

\section{INTRODUCTION}

Magnetic semiconductors are characterized by the presence of both charge carriers and magnetic moments. The interaction between them leads to spindisorder scattering of the charge carriers i.e. there is a strong influence of magnetic properties on the transport properties of magnetic semiconductors ${ }^{[1]}$. Also, the temperature has great effects on the electrical properties of the magnetic semiconductors. One of these effects is the change of the activation energy at the magnetic transition from ordered to disordered states has been reported for many ferrites ${ }^{[2-5]}$. In fact there are many points of view to interpret such a change in the activation energy. El-Hiti ${ }^{[6]}$ attributed this change to the randomization of the magnetic moments after the Curie temperature $\mathrm{T}_{\mathrm{c}}{ }^{[6]}$. Moreover, it was found that the difference between the activation energy in the paramagnetic region and that in the ferromagnetic region increases monotonically with the Curie temperature. On the other hand, this change was ascribed to a volume expansion of the sample at $T_{c}{ }_{c}^{[7,8]}$. Further point of view for this change is the formation of the magnetic spin polaron (electron- magnetic moment interaction) ${ }^{[9]}$. It was found that the formation of the magnetic spin polaron is most probable after the Curie temperature.

To determine the most probable mechanism for the change of the activation energy at $\mathrm{T}_{C}, \mathrm{Mn}-\mathrm{Zn}$ ferrite was chosen as it is one of the most important ferrites from the technological point of view. It has been used in many devices such as magnetic recording inductor cores, electromagnetic wave absorbers, converters etc.

Thus, in this work, the effect of both the magnetic field and the temperature on the electrical resistivity and activation energy are studied.

\section{MATERIALS AND METHODS}

Preparation of the sample: $\mathrm{Mn}-\mathrm{Zn}$ ferrite sample, with the chemical formula $\mathrm{Mn}_{0.6} \mathrm{Zn}_{0.4} \mathrm{Fe}_{2} \mathrm{O}_{4}$, was prepared by conventional ceramic method. High purity oxides of $\mathrm{ZnO}$ and $\mathrm{Fe}_{2} \mathrm{O}_{3}$ with $\mathrm{MnCO}_{3}$ were mixed together according to their molecular weights. The mixture was ground to very fine powder and presintered at $900^{\circ} \mathrm{C}$ for $15 \mathrm{hrs}$. The presintered mixture was ground again and pressed at room temperature under a pressure $3.8 \times 10^{8} \mathrm{~Pa}$ into tablet shape for the electrical measurements. Finally the sample was sintered at $1300^{\circ} \mathrm{C}$ for $4 \mathrm{hrs}$ in two cycles and then slowly cooled in $\mathrm{N}_{2}$ atmosphere to room temperature at the rate $1^{\circ} \mathrm{C} / \mathrm{min}$.

X-ray and porosity measurements: X-ray diffraction pattern was performed using a diffractometer of type X'Pert Graphics and identified with $\mathrm{Cu}_{\alpha}$ radiation.

The porosity percentage $(\mathrm{P} \%)$ was determined, using Archimedes' principle, by the formula $\mathrm{P} \%=(1-$ $\left.d / d_{x}\right)$, where, $d$ is the real density of the sample and $d_{x}$ is the theoretical $\mathrm{x}$-ray density $\left(\mathrm{d}_{\mathrm{x}}=8 \mathrm{M} / \mathrm{Na}^{3}\right.$ where, $\mathrm{M}$ is the molecular weights and $\mathrm{N}$ is Avogadro's number). The porosity of the prepared sample was found to be about $6 \%$. The electrical resistivity is measured at different magnetic field strength $(0.0-0.9 \mathrm{~T})$ by using two probe method. To eliminate Hall effect, the direction of the magnetic field was parallel to the direction of the electric current.

\section{RESULTS AND DISCUSSION}

X-ray diffraction: Figure 1 shows the $x$-ray chart of the investigated sample. By comparison this chart with the JPDS card of Mn-Zn ferrite, single spinal phase formation is confirmed. 


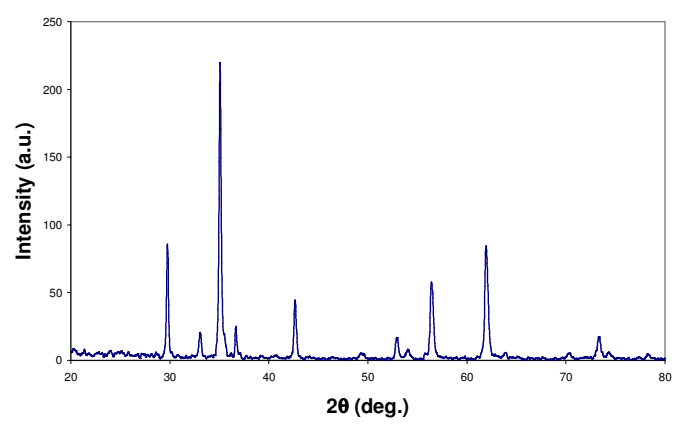

Fig. 1: X-ray diffraction pattern of $\mathrm{Mn}_{0.6} \mathrm{Zn}_{0.4} \mathrm{Fe}_{2} \mathrm{O}_{4}$

The lattice parameter of the investigated sample could be determined using Bragg's law. The lattice parameter, $\mathrm{a}$, was found to be about $8.584 \mathrm{~A}^{\circ}$ which is in a good agreement with that reported previously $\left(8.623 \mathrm{~A}^{\mathrm{o}}\right)^{[10]}$

Effect of application of magnetic field on the electrical resistivity: Figure 2 shows the dependence of the electrical resistivity on the magnetic field $(\mathrm{H})$ at different temperatures. It is clear that, there is an inverse relation between the electrical resistivity and the magnetic field. This could be simply explained through the scattering of the charge carriers (electrons) with the magnetic moments. Such a scattering increases with increasing the randomization of the magnetic moment ${ }^{[1]}$. However, the application of the magnetic field leads to the magnetic ordering to increase. Thus, the scattering of the charge carriers decreases and hence the resistivity decreases.

Furthermore, it is obvious that, the decrease of the electrical resistivity with the applied magnetic field could be divided into two stages. The value of the transient point between the two stages shifts toward to lower magnetic field as the temperature increases (Fig. 2a). For temperatures higher than the Curie temperature the transient point completely disappears as shown in Fig. 2d. This could be attributed to the following reasons:

In the $1^{\text {st }}$ stage, low magnetic field region, the magnetic ordering arises from the easy rotation moments (Domain wall), which lead to decrease electrical resistivity.

In the $2^{\text {nd }}$ stage, high magnetic field region, further increase in the magnetic ordering takes place by the hard rotation moments (domain rotation) and so further decrease in the electrical resistivity takes place. In the same time, as the temperature increases the magnetic anisotropy field decreases and so the domain wall thickness increases on account of the domain size ${ }^{[11]}$. Therefore, the domain wall ordering becomes more effective on the electrical resistivity than that of the domain ordering. Moreover, the decrease of the exchange interaction between the magnetic moments by increasing the temperature leads to decrease the magnitude of the magnetic field required to rotate them.
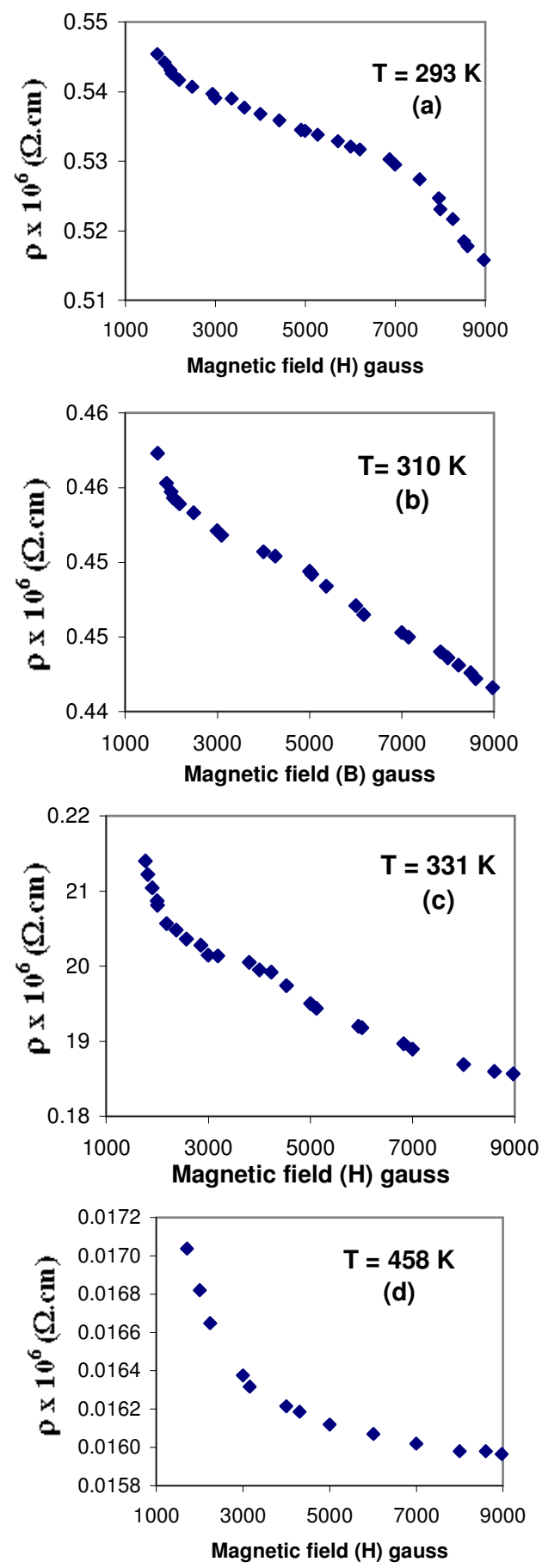

Fig. 2: Variation of resistivity $(\rho)$ with the applied magnetic field of $\mathrm{Mn}_{0.6} \mathrm{Zn}_{0.5} \mathrm{Fe}_{2} \mathrm{O}_{4}$

For temperatures higher than the Curie temperature, there are no domains and so the ordering arises only from the rotation of the random magnetic moments i.e. there is only one stage as shown in Fig. $2 \mathrm{~d}$.

The effect of temperature on the electrical resistivity: Figure 3 shows the variation of the electrical resistivity, $\log (\rho)$ with temperature 


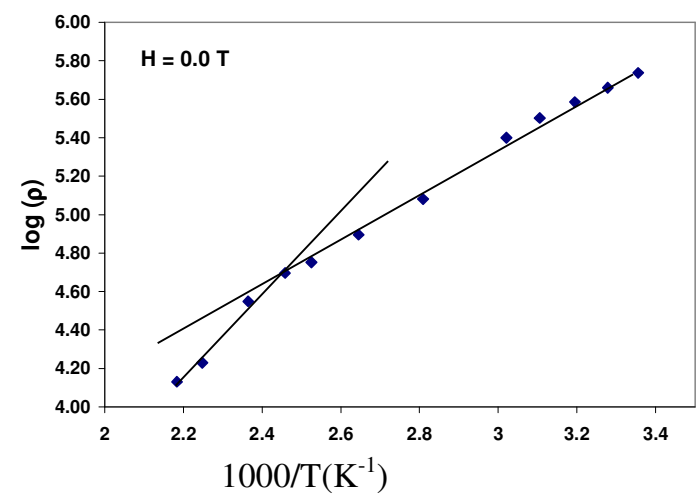

Fig. 3:Temperature dependence of $\log (\rho)$ of $\mathrm{Mn}_{0.6} \mathrm{Zn}_{0.4} \mathrm{Fe}_{2} \mathrm{O}_{4}$ in the absence of magnetic field

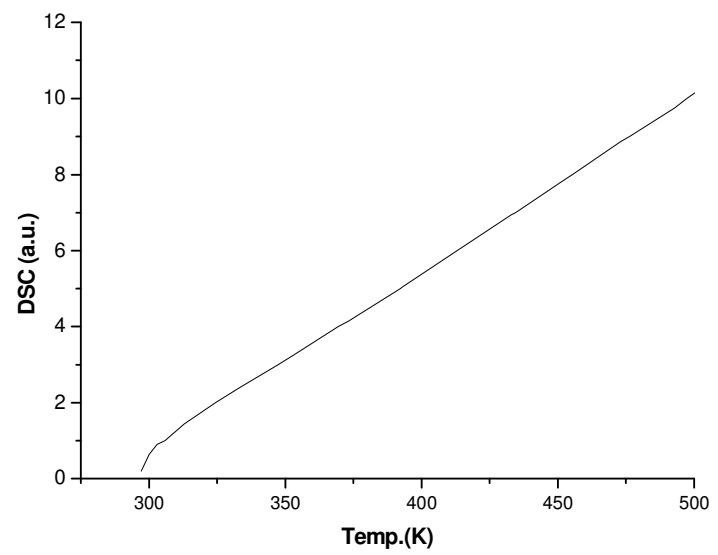

Fig. 4:Temperature dependence of DSC

represented as $1000 / \mathrm{T}\left(\mathrm{K}^{-1}\right)$ for $\mathrm{Mn}-\mathrm{Zn}$ ferrite in the absence of the magnetic field. It is obvious that, the electrical resistivity decreases almost linear with increasing the temperature. Also, there is a change in activation energy at about $413 \mathrm{~K}$ which corresponding to the curie temperature determined from the magnetic measurements $(402 \mathrm{~K})^{[12]}$ and $(393 \mathrm{~K})^{[13]}$. Moreover, it is clear that, the activation energy in the paramagnetic region $\left(\mathrm{E}_{\mathrm{para}} \approx 0.44 \mathrm{ev}\right)$ is greater than that in the ferrimagnetic one $\left(\mathrm{E}_{\text {ferri }} \approx 0.26 \mathrm{ev}\right)$. This increasing in the activation energy was attributed to the volume expansion during the magnetic transition form orderdisorder state ${ }^{[7,8]}$. To confirm this assumption, DSC measurement was performed from room temperature up to above the Curie temperature where, it is known that, the volume expansion should be associated with an endothermic peak in the DSC chart of the investigated sample. Figure 4 shows DSC chart. It is obvious that, there is a good thermal stability of the sample. Therefore, the assumption that the increase of the activation energy is due to the volume expansion should be excluded. Similar result was reported for $\mathrm{Ni}-\mathrm{Zn}$ ferrite $^{[10]}$.

In the following, the spin polaron model will be used to explain the increase in the activation energy.
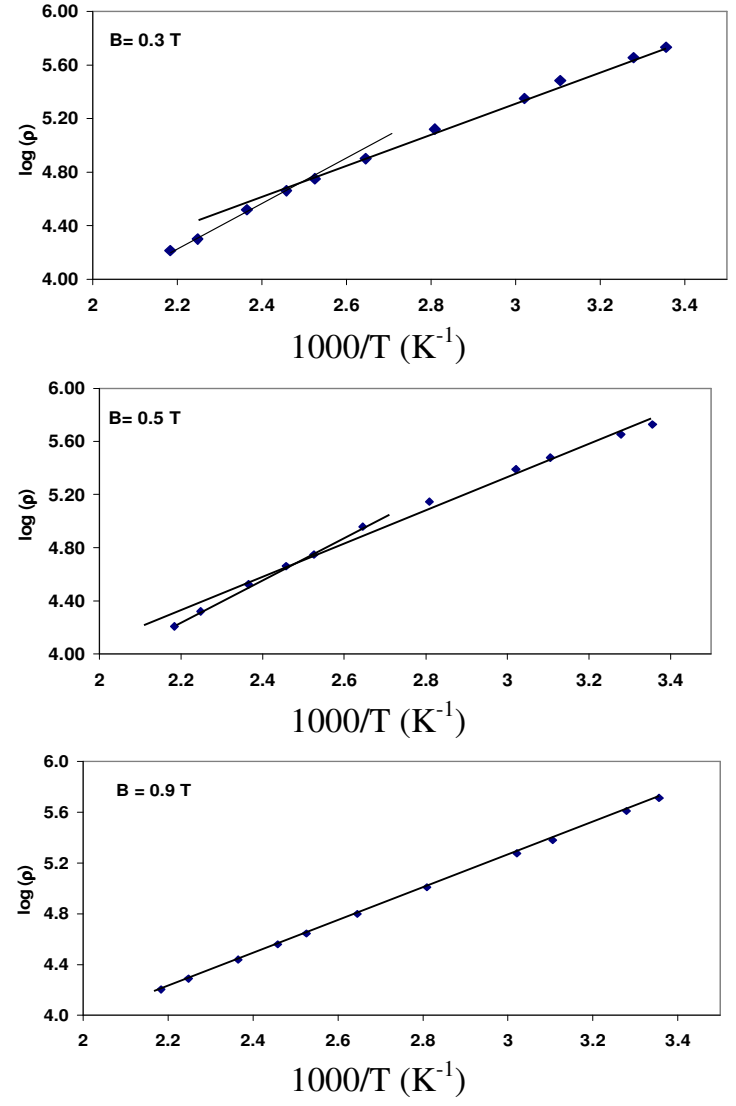

Fig. 5: Temperature dependence of $\log (\rho)$ of $\mathrm{Mn}_{0.6} \mathrm{Zn}_{0.4} \mathrm{Fe}_{2} \mathrm{O}_{4}$ in the presence of magnetic field

According to this model, the total energy of the spin polaron is given by ${ }^{[9]}$.

$E=\frac{5 \hbar^{2} \pi^{2}}{6 m^{*}}\left(\frac{4 m J_{2}}{\hbar^{2} \pi a^{3}}\right)^{2 / 5}-J_{1}$

where, $\mathrm{m}^{*}$ is the electronic mass, a is the lattice parameter, $J_{1}$ is the exchange energy between the spins of the conduction electrons and the ionic spins and $\mathrm{J}_{2}$ is the exchange energy between the ion spins themselves. In fact at temperature $T<T_{c}$, the magnetic moments of the ions are parallel and so, the exchange energy between the ionic spins is much greater than that between the conduction electrons and the ionic spins. On the other hand, at $\mathrm{T}>\mathrm{T}_{\mathrm{c}}$, the magnetic moments of the ions become randomly oriented and so, it can be easily polarized by the spin of the conduction electron i.e. $\mathbf{J}_{1}$ dominates $\mathbf{J}_{2}$. Thus, the electron drags the spin polarization and hence its effective mass increases. Therefore, the activation energy increases. Accordingly the activation energy of the spin polaron $\left(\mathrm{E}_{\mathrm{s}}\right)$ could be suggested to be $\left(\mathrm{E}_{\mathrm{para}}-\mathrm{E}_{\text {ferri }}\right)$.

The effect of the application of an external magnetic field on the activation energy will give further confirmation for this assumption. 


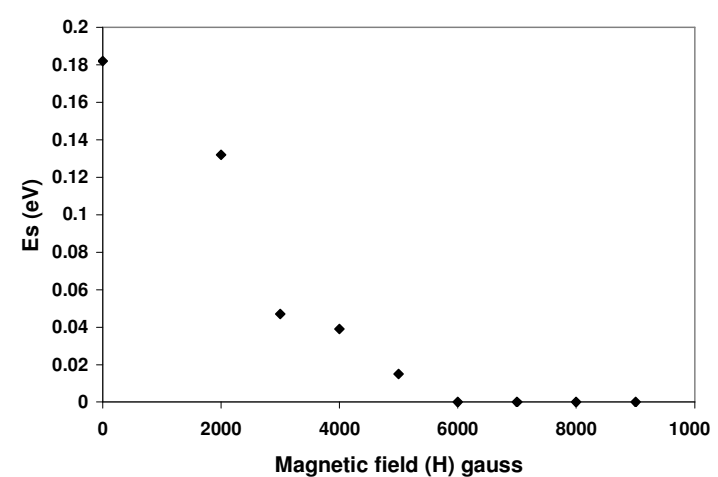

Fig. 6: Effect of magnetic field $(\mathrm{H})$ on the activation energy of the spin polaron

The effect of the magnetic field on the activation energy: Figure 5 shows the relation between $\log (\rho)$ and $1000 / \mathrm{T}\left(\mathrm{K}^{-1}\right)$ at three selected magnetic fields $(0.3$ $\mathrm{T}, 0.5 \mathrm{~T}$ and $0.9 \mathrm{~T})$. It is found that for temperatures lower than $\mathrm{T}_{\mathrm{C}}$, the slope of the curves, $\mathrm{E}_{\mathrm{Ferri}}$, is almost independent of the magnetic field. On the other hand, for temperatures higher than $T_{C}$, the slope of the curves, $\mathrm{E}_{\mathrm{para}}$, decreases with increasing the magnetic field such that for fields $\geq 0.6 \mathrm{~T}$ there is no change in the activation energy.

The dependence of the spin polaron activation energy $E_{s}\left(E_{s}=E_{\text {para }}-E_{\text {ferri }}\right)$ on the applied magnetic field is shown in Fig. 6. It is clear that, as the applied field increase the activation energy of the spin polaron decreases. For magnetic field $\geq 0.6 \mathrm{~T}, \mathrm{E}_{\mathrm{s}}$ tends to zero i.e. there is no effect of the spin polaron on the activation energy.

This means that, the application of the magnetic field has a great effect on the formation of the spin polaron.

These results could be explained as follows: As it was discussed above, there are two types of exchange interactions $\mathbf{J}_{1}$ and $\mathbf{J}_{2}$. In the presence of an external magnetic field another interaction between this applied field and the ion spins, $\mathrm{J}_{3}$, should be taken into consideration.

In the ferrimagnetic region, the interaction between the spin moments $\left(\mathrm{J}_{2}\right)$ dominates those of $\mathrm{J}_{1}$ and $\mathrm{J}_{3}$ which explains the constancy of $E_{\text {ferri }}$ with the magnetic field. In the paramagnetic region and the absence of the magnetic field, $\mathrm{J}_{2}$ vanishes while $\mathrm{J}_{1}$ increases and the formation of the spin polaron takes place. By increasing the applied magnetic field, the interaction between the magnetic field and the ion spins $\left(J_{3}\right)$ increases on the account of that between the spin of the conduction electrons and the ion spins, $\mathrm{J}_{1}$. It seems that the applied magnetic field acts to reduce the number of ion spins sharing in the spin polaron. This leads to a decrease of the effective mass of the conduction electron and so decreases its activation energy. At high magnetic field, the interaction between the applied field and the ionic magnetic moments $\left(J_{3}\right)$ dominates the interaction between conduction electrons and these moments. This means that, there is no a chance for the formation of the spin polaron i.e. $\mathrm{E}_{\mathrm{s}}=$ zero. Thus there is no difference between $\mathrm{E}_{\text {ferri }}$ and $\mathrm{E}_{\text {para }}$.

\section{CONCLUSION}

The electrical resistivity of Mn-Zn ferrite has an inverse relation with the applied magnetic field. This was attributed to the magnetic ordering which reduces the scattering of the charge carriers by the disordered spins. In Mn- $\mathrm{Zn}$ ferrite there is no volume expansion during the magnetic transition from the ferrimagnetic to paramagnetic region. The increase of the activation energy in the paramagnetic region is due to the formation of magnetic spin polaron, which increases the effective mass of the conduction electron. The evidence of the formation of the magnetic spin polaron is the great effect of application of an external magnetic field on the activation energy in the paramagnetic region. The control of the effective mass of the charge carriers by the application of an external magnetic field is very important for the fabrication of the changeable spin magnetic transistors. It seems that, this material has a great advantage for the research in this point.

\section{REFERENCES}

1. Haas, C., 1970. Spin-disorder scattering and band structure of the ferromagnetic Chalcogenide Spinel. IBM J. Res. Develop., pp: 282.

2. Rosenberg, M., P. Nicolau and I. Bunget, 1966. On the electrical properties of some iron-copper and coppermanganese ferrites. J Phys. Stat. Sol., 15: 512.

3. Ghani, A.A., A.I. Eatah and A.A. Mohamad, 1980. Phase transition and conductivity in $\mathrm{Cu}-\mathrm{Ni}$ ferrites. Proc. ICF3, Japan, pp: 216.

4. Mazen, S.A. and M.A Ahmed, 1982. The deficiency effect of iron on the conduction mechanism of CO-Znferrites. Phys. Stat. Sol. A, 73: K307.

5. Satyanarayana, R. and S.R. Murthy, 1984. Electrical conductivity of barium-strontium ferrites. Phys.Stat. Sol. A, 84: 655 .

6. El-Hit, 1995. Electrical resistivity at the curie point for some mixed ferrites. Phys. Stat. Sol. A, 147: K25.

7. Zemansky, M.W., 1968. Heat and Thermodynamics. MC Graw Hill, NewYork, pp: 460.

8. Sattar, A.A., A.H. Wafik and H.M. El-Sayed, 2001. Transport properties of trivalent substituted Li-ferrites. J. Mat. Sci., 36: 4703.

9. Mott, N.F. and E.A. Davis, 1971. Electronic Processes in non Crystalline Materials. Clarendon Press Oxford, pp: 175.

10. Sattar, A.A., H.M. El-Sayed and M.M. El-Tabey, 2005. The effect of al-substitution on structure and electrical properties of Mn-Ni-Zn ferrites. J. Mat. Sci., 40: 4873.

11. Chikazumi, S. and S. Charap, 1964. Physics of Magnetism. John Wiley and Sons, New York.

12. Sattar, A.A., H.M. El-Sayed, K.M. El-Shokrofy and M.M. El-Tabey, 2005. Effect of manganese substitution on the magnetic properties of nickel-zinc ferrite. JMEPEG, 14: 99.

13. Hitaro, K., T. Aoyamoy, M. Yoshinaka and O. Yanguchi, 1999. Microstructures and magnetic properties of low- temp. Sintering $\mathrm{Mn}-\mathrm{Zn}$ ferrites without and with addition of Lithium-Borate glass. J. Mag. Mag. Mat., 205: 283. 